strategies adopted by Saudi healthcare workers to alleviate the stress related to COVID-19 pandemic.

Method. A descriptive cross-sectional study was performed in the period between September and November/2020. A sample of 381 healthcare workers (Physicians, nurses, and technicians) were recruited from King Saud Medical City (KSMC). Both Perceived Stress Scale-4 (PSS-4) and BRIEF-COPE scale were used to assess the levels of stress and the stress coping strategies, respectively. Descriptive statistics were used to analyze the healthcare workers' responses about the COVID-19 related stress and their adopted coping strategies

Result. The results of the study showed that there was a moderate to high level of COVID-19 related stress $(11.64 \pm 0.73)$ among the Saudi healthcare workers. In addition, it was found that planning $(3.89 \pm 0.61)$, positive reframing (3.69 \pm 0.77$)$, venting (3.39 \pm $1.01)$, and emotional support $(3.27 \pm 0.63)$ were the most adopted coping strategies by the healthcare workers to overcome and reduce the stress levels

Conclusion. The study concluded that both problem-focused and emotion-focused stress coping strategies were the most commonly adopted coping strategies among Saudi healthcare workers in KSMC. The study recommends increasing the number of the healthcare workers in the KSMC, in addition to increasing the healthcare workers' knowledge, awareness and practice of the stress coping strategies, especially in crisis events, such as COVID-19 pandemic.

\section{Psychiatric comorbidities with autism spectrum disorder in an adult clinic sample}

Rifat Binte Radwan ${ }^{1 \star}$ and Chiro Islam Mallik ${ }^{2}$

${ }^{1}$ Royal Victoria Infirmary, Cumbria, Northumberland, Tyne and Wear NHS Foundation trust and ${ }^{2}$ Community Learning Disability Team, Hertfordshire Partnership University NHS Foundation Trust ${ }^{*}$ Corresponding author.

doi: 10.1192/bjo.2021.639

Aims. As part of continuity, prevalence of Autism Spectrum Disorder (ASD) is nearly the same in adults as children and is associated with other comorbid psychiatric disorders that have substantial impact on their life and complex the intervention. This study aimed to examine psychiatric comorbidity in referred adult ASD patients compared to non-ASD psychiatric patients. It has been hypothesized that comorbid psychiatric disorders were higher among patients with ASD than patients without ASD.

Method. In total, 36 adults with ASD referred in the year 2019 in a psychiatric consultation center in Dhaka city were included in the study. They were derived from the case register of the center. Similar number of age and sex-matched adult psychiatric patients without ASD were selected for comparison. All patients were referred for psychiatric consultation. Socio-demographic variables were collected from the patients' record. Diagnosis of psychiatric disorders including ASD was made by an experienced psychiatrist. It was done clinically based on all available information, examination and relevant investigations. Diagnoses were assigned according to DSM-5. Then comparisons of psychiatric disorders were made between the two patient groups.

Result. The cases were ranged from 18-41 years with the mean of $26.72 \pm 6.5$ years. Among them, 22 were male and 14 were female. Male-female ratio was 1.6:1. Most of the subjects received no education and were from middle income family with urban background. Mean number of comorbid psychiatric disorders was 1.92 in patients with ASD and 1.67 in patients without
ASD and the difference was significant $(\mathrm{P}=0.04)$. Most two frequent comorbidities among ASD patients were Obsessive Compulsive Disorder (27.77\%) and Major Depressive Disorder (25\%) followed by Specific Phobia(19.44\%), Social Phobia and Intermittent Explosive Disorder(16.67\%) for each, Attention Deficit Hyperactivity Disorder(13.89\%) and Conduct Disorder $(11.11 \%)$. All these disorders were significantly higher than patients without ASD. Conversely, Major Depressive Disorder (30.55\%) was most frequent among the patients without ASD and that was even significantly higher than patients with ASD. Other frequent disorders like Bipolar Disorder, Schizophrenia, Generalized Anxiety Disorder and Substance Related Disorder were also higher among non-ASD patients.

Conclusion. This research shows that comorbid psychiatric disorders were frequently found in patients with ASD. Subsequent broad-based studies using extensive measures of psychopathology are required to confirm these preliminary findings. Greater understanding of the presence of other psychiatric disorders in ASD patients will turn this awareness into action.

\section{Bournewood in Belfast: who's ready?}

Hayley Bowes*, Joseph Kane and Gillian McPherson

Belfast Health and Social Care Trust

${ }^{\star}$ Corresponding author.

doi: $10.1192 /$ bjo.2021.640

Aims. We aimed to determine doctors' confidence in completing capacity assessments and reports associated with new Deprivation of Liberty Safeguards (DoLS), and the impact that these would have on practice. We hypothesised that psychiatrists would have greater confidence in completing the requisite assessments and would anticipate a greater impact on practice than doctors in other specialties.

Background. On the 2ndDecember 2019 DoLS became the first part of the Mental Capacity Act (Northern Ireland) 2016 to be implemented, believed to apply to 7500 individuals across Northern Ireland (NI). As arguably the most significant change in mental health legislation in NI since 1986, the Department of Health commissioned training for all clinicians.

Method. We conducted a cross-sectional survey among doctors working within psychiatry, general medicine, anaesthetics and surgery in Belfast Health and Social Care Trust prior to implementation. The survey comprised seven questions with a 10 -point Likert scale. Statistical analysis included Pearson's $\chi 2$ and Spearman's rank tests.

Result. 79 doctors in psychiatry and 25 in other medical specialties completed the survey.Respondents were moderately confident in completing capacity assessments (median 6 (3-9)) and medical reports (median 5 (1-9)). Those that had completed training $(\mathrm{n}=86 ; 83 \%)$ were significantly more confident in capacity assessment (median $7(2-10)$ vs $4(1-7) ; \chi 2(18)=36.8, \mathrm{p}<0.01)$ and medical report completion (median 5 (1-9) vs 1 (1-5); $\chi 2(16)=27.2, \mathrm{p}=.04)$ than those that had not $(\mathrm{n}=18 ; 17 \%)$. Psychiatrists had greater confidence in conducting capacity assessments (median 7(2-10)) than other doctors (median 5(1-9); $\chi 2(9)=18.2, p=0.04)$. No significant differences were observed between the two groups with respect to medical report completion, or anticipated impact on practice.

Respondents who most frequently conducted capacity assessments as part of their current practice anticipated higher degrees of impact on their individual practice ( $\mathrm{rs}=0.51, \mathrm{p}<0.01)$ and their service $(\mathrm{rs}=0.50, \mathrm{p}<0.01)$ 